\begin{tabular}{|c|c|}
\hline Title & Reversibly tunable helicity induction and inversion in liquid crystal self-assembly by a planar chiroptic trigger molecule \\
\hline Author(s) & Mathews, Manoj; Tamaoki, Nobuyuki \\
\hline Citation & $\begin{array}{l}\text { Chemical Communications, 24, 3609-3611 } \\
\text { https://doi.org/10.1039/0905305k }\end{array}$ \\
\hline Issue Date & $2009-06-28$ \\
\hline Doc URL & http://hdl.handle.net/2115/43143 \\
\hline Rights & Chem. Commun., 2009, 3609-3611 - Reproduced by permission of The Royal Society of Chemistry (RSC) \\
\hline Type & article (author version) \\
\hline File Information & CC24_3609-3611.pdf \\
\hline
\end{tabular}

Instructions for use 


\title{
Reversibly tunable helicity induction and inversion in liquid crystal self- assembly by a planar chiroptic trigger molecule
}

\author{
Manoj Mathews ${ }^{a}$ and Nobuyuki Tamaoki* ${ }^{b}$ \\ Received (in $X X X, X X X) X$ th $X X X X X X X X X 200 X$, Accepted Xth $X X X X X X X X X 200 X$ \\ ${ }_{5}$ First published on the web Xth $X X X X X X X X X 200 X$ \\ DOI: 10.1039/b000000x
}

\begin{abstract}
Reversible control of the helical pitch length and inversion of its helical handedness in an induced cholesteric liquid crystal phase was accomplished via a combination of photochemical and 10 thermal isomerizations of a planar chiral azobenzenophane molecule.
\end{abstract}

Controlling helicity induction and its inversion in artificial systems at different hierarchial levels of molecular selfassembly by external stimuli such as chiral dopants, ${ }^{1}$ 15 temperature $^{2}$ and light $^{3}$ offers potential applications in assymetric synthesis, ${ }^{4}$ sensing, ${ }^{5}$ and memory devices. ${ }^{6}$ Chiral liquid crystal (LC)molecules are well known to self-assemble into many technologically important helical phase structures. ${ }^{7}$ The most well known LC phase having helical structure is 20 cholesteric $\left(\mathrm{N}^{*}\right)$, in which the average direction of molecular axes rotates around a helical axis. Cholesteric phase reflect selectively the circularly polarized fraction of incident light satisfying the Bragg condition, $\lambda=n P$, where $\lambda$, $\mathrm{n}$ and $P$ are wavelength, mean refractive index and helical pich, 25 respectively. Therefore, when its helical pitch $(P)$ is comparable with the wavelengths of visible light, we see reflection colors from the cholesteric LC phase. More interestingly the helical pitch length can be designed to respond to various external perturbations such as dopants,

30 temperature, light and electric fields to revesibly tune the reflected colors for various applications in modern color information technology. ${ }^{8}$ Although, enantiomers of a chiral dopant are known to induce an equal but opposite twist in the cholesteric phase, a reversible switching of the helical 35 handedness by light and temperature proved to be a challenging task.

A chiral photoresponsive dopant can act as both a chiral agent to induce a chiral nematic phase in a nematic liquid crystal and a photoresponsive moiety to control the helical ${ }_{40}$ pitch through photo-isomerization. Considerable progress have been made towards the photo modulation of the helical pitch length by the design of dopants incorporating photoresponsive moieties such as azobenzenes, fulgides, diarylethenes, spiropyrans, and overcrowded alkenes with ${ }_{45}$ point, axial, and helical chirality. ${ }^{9}$ However, chiral switchable dopants with ability to induce cholesteric helix of opposite sign for both switch states remains rarely known. ${ }^{10,11}$

Over the past decade, we have been exploring the strategies to address the reversible cholesteric reflection color control 50 over the entire visible range for applications in optically rewrittable memory devices. ${ }^{8,} 12$ Recently, we reported the first example of a photoresponsive planar chiral dopant in the form of a monocyclic azophane and successfully achieved a fast photon mode reversible full-range color control in ${ }_{55}$ induced cholesterics. ${ }^{13}$ Here we report our success in reversibly controlling the helical pitch length as well as the handedness of the induced helix by photochemical and thermal isomerizations of a planar chiral bicyclic azobenzenophane (1) in host nematic LCs.

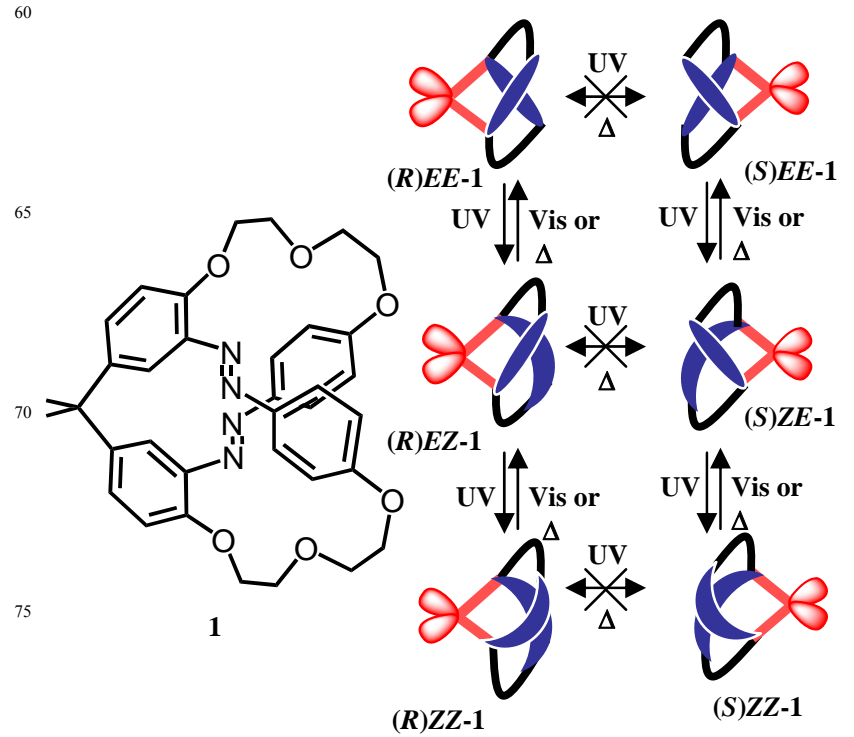

80 Fig.1 Structure of planar chiral bicyclic azobenzenophane (1) and the schematic representation of photochemical and thermal processes taking place in its $R$ and $S$ enantiomers. Blue structure in the scheme represent photochromic azobenzene unit.

Target molecule was synthesized as pure trans isomer of the 85 racemic compound $E, E-1$ and the structure was fully characterized by NMR and X-ray crystallographic analysis. ${ }^{14}$ The restricted "flip-flop" of the 2,2-diphenylpropane unit through the cyclophane cavity (Fig. 1) impart an element of planar chirality to the molecule. Chiral HPLC analysis 90 confirmed the existance of a pair of enantiomers. We resolved racemic E,E-1 through preparative HPLC using a chiral column. The enantiomer that eluted first, which we assigned as (S)E,E-1, exhibited CD bands at 230, 350, and $455 \mathrm{~nm}$ with $\Delta \varepsilon$ of $+78,+70$ and $-65 \mathrm{M}^{-1} \mathrm{~cm}^{-1}$ respectively. As 95 expected, the second eluted enantiomer $(\boldsymbol{R}) \boldsymbol{E}, \boldsymbol{E}-\mathbf{1}$ shows a complete mirror image CD spectrum of its enantiomer (Fig. 2). In solution, E,E-1 undergoes efficient cis-trans isomerizations by UV and visible light irradiations (Fig. 3). Absorption spectra exhibit distinct bands for $\pi-\pi^{*}$ and $n-\pi^{*}$ 




Fig.2 CD spectra of enantiomers of $\mathbf{1}$; (S)E,E-1 (blue line) and $\boldsymbol{( R ) E , E - 1}$ (red line) in $\mathrm{CH}_{3} \mathrm{CN}$.

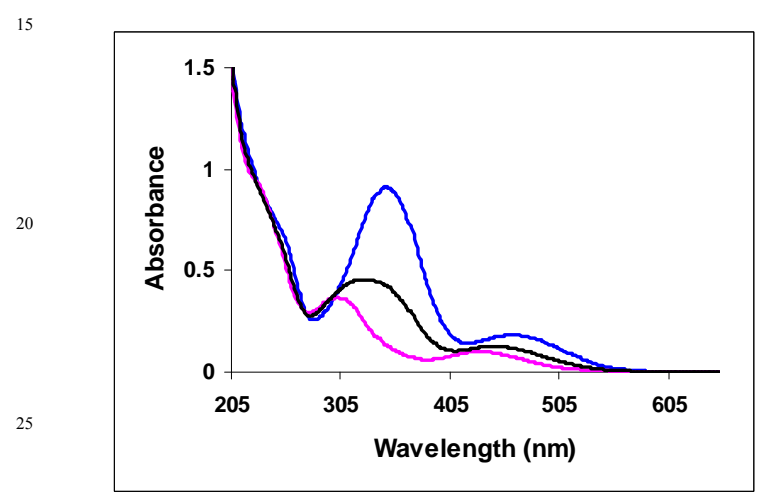

Fig.3 Absorption spectral change of $\boldsymbol{E , E - 1}$ in acetonitrile upon irradiation at room temperature: (a) blue line, initial state before 30 irradiation; (b) red line, photostationary state (PSS) after irradiation at $365 \mathrm{~nm}$; and (c) black line, PSS after irradiation at $436 \mathrm{~nm}$.

transitions of the azochromophore. Upon UV irradiation $\left(\lambda_{\max }\right.$ $=365 \mathrm{~nm})$ due to photochemical trans $(E, E-1)$ to cis $(E, Z-1$

35 and $Z, Z-1)$ isomerization, there is a gradual decrease seen in the $\pi-\pi^{*}$ transition bands in the absorption spectra with concomitant increase in the $\mathrm{n}-\pi^{*}$ band around $450 \mathrm{~nm}$. Photostationary state (PSS) was reached after UV irradiation for about $60 \mathrm{~s}$. We determined the ratio of trans:cis isomers in 40 the $\mathrm{PSS}_{\mathrm{UV}}$ as $87 \%(Z, Z-1), 12 \%(E, Z-1)$ and $1 \%(E, E-1)$ by HPLC analysis. On visible light irradiation $\left(\lambda_{\max }=436 \mathrm{~nm}\right)$ reverse absorption changes occurred and a $\operatorname{PSS}_{\mathrm{vis}}$ was obtained in about $90 \mathrm{~s}$. The relative concentrations of the $E, E$, $E, Z$ and $Z, Z$ isomers in the $P_{S S}$ vis were $23 \%, 47 \%$ and $30 \%$ 45 respectively. By chiral HPLC analysis we confirmed that racemization between the enantiomers does not occur either thermally or photo-chemically. In other words, the size of the cyclophane cavity formed for $E, E$, and $Z, Z$ isomers are shallow enough to prevent the racemization. On the contray, ${ }_{50}$ its higher homologue obtained by increasing the chain length of ethylene glycol unit resulted in photo-induced racemization through its $Z, Z$ isomer. ${ }^{14}$ Fig. 1 summarizes the isomerization characteristics of $(\boldsymbol{S}) \boldsymbol{E}, \boldsymbol{E}-\mathbf{1}$ and $(\boldsymbol{R}) \boldsymbol{E}, \boldsymbol{E}-\mathbf{1}$ enantiomers in solution, highlighting their photochemically and thermally 55 reversible isomerization to $(\boldsymbol{S}) \boldsymbol{E}, \boldsymbol{Z}-\mathbf{1},(\boldsymbol{S}) \mathbf{Z}, \mathbf{Z}-\mathbf{1}$ and $(\boldsymbol{R}) \boldsymbol{E}, \boldsymbol{Z}-\mathbf{- 1}$, $(R) Z, Z-1$ respectively without undergoing any racemization.

Next we investigated the potential of $(\boldsymbol{R}) \boldsymbol{E}, \boldsymbol{E}-\mathbf{1}$ and $(\boldsymbol{S}) \boldsymbol{E}, \boldsymbol{E}$ 1 as chiral transfer agents in three structuraly different ${ }^{15}$ commercially available host nematic liquid crystals (NLCs) 60 namely ZLI-1132, DON-103 and 5-CB. As expected, all the LC mixtures induced a chiral $\mathrm{N}$ phase which was evidenced as a fingerprint texture ${ }^{16}$ under polarized optical microscope. When a chiral solute is dissolved in nematic liquid crystal at the limit of low concentration, the induced pitch $P$ is ${ }_{65}$ correlated with the weight concentration $C_{w}$ of the dopant (weight of dopant / weight of host NLC) and its enantiomeric putiry $r$ according to the equation $P^{-1}=\beta C_{w} r$. The proportionality constant $\beta$ is referred to as the helical twisting power (HTP). The induced helical pitch and its thermal and 70 photoresponsive behavior was measured using Cano wedge cells. ${ }^{16}$ The corresponding change in HTP values were calculated based on above mentioned equation and the results are summarized in Table 1.

Table 1. Helical twisting power $\left(\beta / \mu \mathrm{m}^{-1}\right)$ of dopants in different NLC 75 hosts as determined by Cano's wedge method and the observed change in values by irradiation ${ }^{\mathrm{a}}$.

\begin{tabular}{|c|c|c|c|c|c|}
\hline \multirow[t]{2}{*}{ Dopant } & Host & \multicolumn{3}{|c|}{$\beta\left(\mu \mathrm{m}^{-1}\right)$} & $\triangle \beta[\%]^{b}$ \\
\hline & NLC & Initial & $\mathrm{PSS}_{\mathrm{uv}}$ & PSS $_{\text {vis }}$ & \\
\hline \multirow[t]{3}{*}{$80(\boldsymbol{R}) \boldsymbol{E}, \boldsymbol{E}-1$} & $5 \mathrm{CB}$ & -6 & +32 & +26 & 633 \\
\hline & ZLI-1132 & -7 & +28 & +23 & 500 \\
\hline & DON-103 & +5 & +37 & +28 & 640 \\
\hline \multirow{2}{*}{ (S)E,E-1 } & $5 \mathrm{CB}$ & +6 & -32 & -26 & 633 \\
\hline & ZLI-1132 & +7 & -28 & -23 & 500 \\
\hline 85 & DON-103 & -5 & -37 & -28 & 640 \\
\hline
\end{tabular}

${ }^{\text {a }}$ Positive and negative values represent right-and left-handed helical twists, respectively. ${ }^{\mathrm{b}}$ Percent change in $\beta$ observed from initial to $\mathrm{PSS}_{\mathrm{UV}}$.

Both dopants in its all trans isomer configuration i.e. (R)E,E1 and $(\boldsymbol{S}) \boldsymbol{E}, \boldsymbol{E}-1$ show very low HTP values in all the three host ${ }_{90}$ NLCs. The helical sense of the induced $\mathrm{N}^{*}$ phase was determined by both contact as well as color shift method. ${ }^{15}$ Chiral dopant (R)E,E-1 in 5CB and ZLI-1132 induced a lefthanded helix, while a right-handed helix was obtained from its enantiomer $(\boldsymbol{S}) \boldsymbol{E}, \boldsymbol{E}-1$. Interestingly, an opposite helix sense 95 was induced by $(\boldsymbol{R}) \boldsymbol{E}, \boldsymbol{E}-\mathbf{1}$ and $(\boldsymbol{S}) \boldsymbol{E}, \boldsymbol{E}-\mathbf{1}$ in DON-103 i.e., a right-handed helix by $(\boldsymbol{R}) \boldsymbol{E}, \boldsymbol{E}-\mathbf{1}$ and a left- handed helix by (S)E,E-1. This could be explained based on the difference in intermolecular associations between dopants and the hosts; 5CB and ZLI-1132 have rigid biaryl moieties and high 100 longitudinal dipole, whereas DON-103 posses flexible ester linkage and transeverse dipole. In the next step we investigated the effect of isomerization of dopants on the pitch values and helix handedness. Photoinduced variations in HTP values and the pitch were directly observed as change in 105 distance between the Cano lines when sample in a wedge cell was observed with a polarized optical microscope under UV or visible light illumination (Fig.4). Duing the process of UV irradiation of LC mixtures containing $1 \mathrm{wt} \%$ of $(\boldsymbol{R}) \boldsymbol{E}, \boldsymbol{E}-\mathbf{1}$ and (S)E,E-1 in 5CB and ZLI-1132, to begin with Cano lines 110 disappeared completely (with in $20 \mathrm{~s}$ of irradiation) before reappearing again on continuing the irradiation to attain the $\mathrm{PSS}_{\mathrm{UV}}$ in about $90 \mathrm{~s}$. The distance between the lines were considerably shortened at PSS $_{\mathrm{UV}}$ pointing to an increase in the HTP and thus shortening of the helical pitch length due to 115 trans-cis isomerization. More interestingly, we found that helicity of the induced $\mathrm{N}^{*}$ phase at $\mathrm{PSS}_{\mathrm{UV}}$ to be opposite to that of the initial state in all the four LC mixtures. 


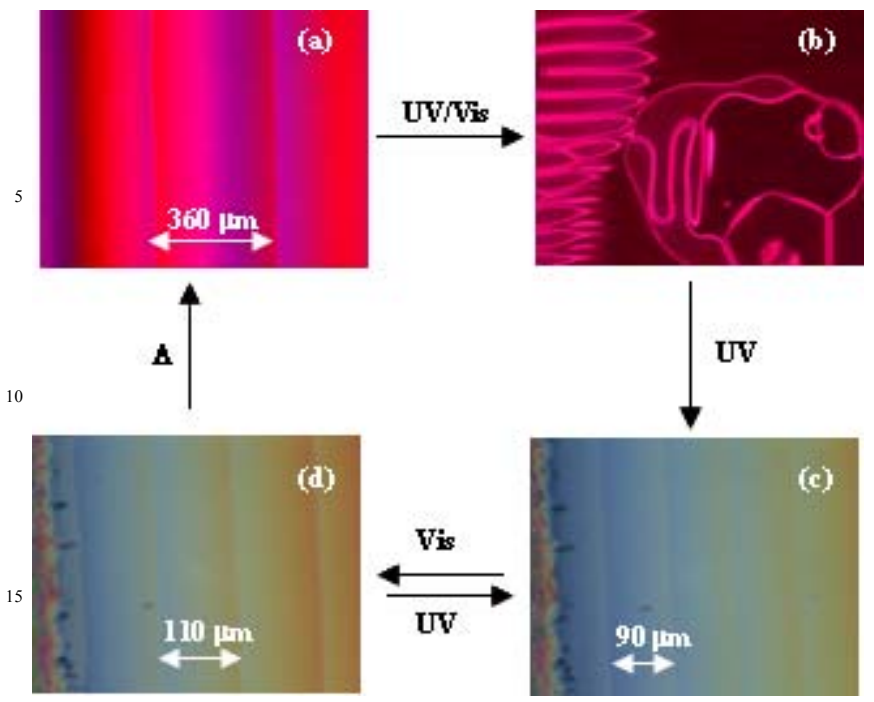

Fig.4 Polarized optical photomicrographs of $1 \mathrm{wt} \%$ of $(\boldsymbol{R}) \boldsymbol{E}, \boldsymbol{E}-\mathbf{1}$ dissolved in ZLI-1132 at RT: Reversible change occurring in pitch by irradiation of 20 sample in a wedge type cell; (a) before irradiation, (b) N phase obtained by exposure of sample to UV or Vis irradiation, (c) $\mathrm{PSS}_{\mathrm{UV}}$, (d) $\mathrm{PSS}_{\mathrm{VIS}}$.

Observation of $\mathrm{N}$ phase due to destabilization of the initially induced helical structures by trans-cis photo-isomerization prior to regeneration of cholesteric helix supported the 25 arguement of helical switching. An increase in distance between the Cano lines were observed upon visible light irradiation of the sample in $\mathrm{PSS}_{\mathrm{UV}}$ induced $\mathrm{N}^{*}$ phase due to the reverse process. However, the Cano lines never disappeared on the course to the $\mathrm{PSS}_{\mathrm{Vis}}$ state and did not 30 result in any change in sign of the cholesteric helicity. This was further confirmed by observing a change in sign of the cholesteric helicity even by visible light irradiation of the initial sample. The effective change in HTP values by alternate visible and UV irradiations were calculated to be $3523 \%$ and $22 \%$ for $5 \mathrm{CB}$ and ZLI-1132 LC mixtures respectively. Helicity reverted to the initial state by allowing the LC mixture to relax back to the thermodynamically favourable trans form in the dark in about 12 hours. Although the presence of two azobenzene moieties make the PSS 40 compositions complex, it is possible to calculate the HTP of $E, Z$ and $Z, Z$ isomers using the known isomeric compositions and values of HTP at PSSs and pure E,E isomer. For example, we obtained HTP values for $(\boldsymbol{R}) \boldsymbol{E}, \mathbf{Z}-\mathbf{1}$ and $(\boldsymbol{R}) \mathbf{Z}, \mathbf{Z}-\mathbf{1}$ in $5 \mathrm{CB}$ as +57 and +22 respectively. These observations clearly 45 demonstrate that photoisomerized $E, Z$ and $Z, Z$ isomers induce an opposite cholesteric helix to that of E,E isomer in $5 \mathrm{CB}$ and ZLI-1132 NLC hosts.

On the contrary, (R)E,E-1 and (S)E,E-1 in DON-103 did not show any switching of helicity by photoisomerization. LC 50 mixtures with these dopants shown significant increase in HTP values with $E, E$ to $E, Z$ and $Z, Z$ photoisomerization. The process was reversed on illumination with visible light and about $40 \%$ effective change in the induced pitch by alternate irradiations was achieved.

55 In summary, bicyclic azophanes with planar chirality was employed as photochromic dopants to induce helicity in $\mathrm{N}$ host LCs. We could reversibly control the helical pitch length and more importantly achieve a change in sign of helicity by external light stimuli. A better understanding of solvent-solute 60 interactions on the chirality transfer is required to explain the observed NLC host dependent helicity switching. More examples of planar chiral photoresponsive molecules are under investigation for applications in light driven arificial systems.

65 This work was supported by a grant-in-aid for science research in a priority area "New Frontiers in Photochromism (No. 471)" from the Ministry of Education, Culture, Sports, Science, and Technology (MEXT), Japan.

\section{Notes and references}

$70{ }^{a}$ Liquid Crystal Institute, Kent State University, Kent, OH 44242, USA.

${ }^{b}$ Research Institute for Electronic Science, Hokkaido University, N20,

W10, Sapporo, Hokkaido 0010020, Japan. Fax: +81-11-7069357; Tel:

+81-11-7069356; E-mail: tamaoki@es.hokudai.ac.jp

$\dagger$ Electronic Supplementary Information (ESI) available: [HPLC traces of 75 the racemates and enantiomers of $\mathbf{1}$, details of pitch and helicity measurements. See DOI: 10.1039/b000000x/

1 M. M. Green, S. Zanella, H. Gu, T. Sato, G. Gottarelli, S. K. Jha, G. P. Spada, A. M. Schoevaars, B. L. Ferringa and A. Teramoto, J. Am. Chem. Soc., 1998, 120, 9810-9817.

2 K. S. Cheon, J. V. Selinger and M. M. Green, Angew. Chem. Int.Ed., 2000, 39, 1482-1485; K. Maeda, H. Mochizuki, M. Watanabe and E. Yashima, J. Am. Chem. Soc., 2006, 128, 7639-7650.

3 D. Pijper, M. G. M. Jongejan, A. Meetsma and B. L. Ferringa, J. Am. Chem. Soc., 2008, 130, 4541-4552; D. Pijper and B. L. Ferringa, Soft Mater., 2008, 4, 1349-1372.

4 K. Akagi, G. Piao, S. Kaneko, K. Sakamaki, H. Shirakawa and M. Kyotani, Science, 1998, 282, 1683-1686.

905 K. Maeda, H. Mochizuki, M. Watanabe and E. Yashima, J. Am. Chem. Soc., 2006, 128, 7639-7650.

6 J. J. D. de Jong, L. N. Lucas, R. M. Kellogg, J. H. van Esch and B. L. Feringa, Science, 2004, 304, 278-281.

$7 \quad$ Chirality in Liquid Crystals; H. -S. Kitzerov and C. Bahr, Ed.; Springer: New York, 2001.

8 N. Tamaoki, Adv. Mater., 2001, 13, 1135-1147; V. A. Mallia and N. Tamaoki, Chem. Soc. Rev., 2004, 33, 76-84.

9 For a recent review see R. Eelkema and B. L. Feringa, Org. Biomol. Chem., 2006, 4, 3729-3745.

100 B. L. Feringa, N. P. M. Huck and H. A. van Doren, J. Am. Chem. Soc., 1995, 117, 9929-9930; R.A. van Delden, N. Koumura, N. Harada and B. L. Feringa, Proc. Natl. Acad. Sci. U.S.A., 2002, 99, 4945-4949.

11 S. Pieraccini, G. Gottarelli, R. Labruto, S. Masiero, O. S. Pandolini and G. P. Spada, Chem. Eur. J., 2004, 10, 56325639; The inversion of sign of helicity in the induced chiral nematic phase was not observed in a different NLC host; R. Eelkema, M. M. Pollard, N. Katsonis, J. Vicario, D. J. Broer and B. L. Feringa, J. Am. Chem. Soc., 2006, 128, 14397-14407.

12 N. Tamaoki, A. Parfenov, A. Masaki, H. Matsuda, Adv. Mater., 1997, 9, 1102-1104; H.Akiyama, V. A. Mallia and N. Tamaoki, Adv. Funct. Mater., 2006, 16, 477-484.

13 M. Mathews and N. Tamaoki, J. Am. Chem. Soc., 2008, 130, 11409-11416.

11514 N. Tamaoki and M. Wada, J. Am. Chem. Soc., 2006, 128, 6284-6285.

15 See the supporting information

16 I. Dierking, Ed.; Textures of Liquid Crystals, Wiely-VCH, Weinheim, 2003. 